EPJ Web of Conferences 49, 15004 (2013)

DOI: $10.1051 /$ epjconf/20134915004

(C) Owned by the authors, published by EDP Sciences, 2013

\title{
Search for heavy resonances, and resonant diboson production with the ATLAS detector
}

\author{
Noam Tal Hod ${ }^{1, a}$ \\ ${ }^{1}$ NIKHEF \\ On behalf of the ATLAS collaboration
}

\begin{abstract}
Heavy resonances decaying into a pair of fundamental particles such as $j j, \ell^{+} \ell^{-}, \gamma \gamma$, and $\ell \nu$, are among the most common features to search for phenomena beyond the standard model (SM). Electroweak boson pair production, such as $W W$ or $Z Z$ with subsequent decays to $\ell \nu \ell^{\prime} \nu^{\prime}$ and $\ell \ell j j$ respectively, is a powerful test of the spontaneously broken gauge symmetry of the SM and can be also used to search for phenomena beyond the SM. There is a wide spectrum of theoretical models predicting these kinds of resonant signatures. This note covers several searches for these new phenomena conducted within ATLAS in 2011 and 2012 for the LHC 7 and $8 \mathrm{TeV}$ center of mass energies respectively. No significant deviations from the SM have been observed and therefore, limits are set on the characteristic parameters of several new physics models. These benchmark models include new heavy $Z^{\prime} / W^{\prime}$ gauge bosons, chiral excitation of the SM weak gauge bosons, $Z^{*} / W^{*}$ Randal-Sundrum and ADD gravitons, Composite models for quarks, e.g. $q^{*}$ with substructure scale $\Lambda$, Quantum black holes, $\mathrm{TeV}^{-1}$ Kaluza-Klein excitation of $\gamma / Z$ and more.
\end{abstract}

\section{Introduction}

This note summarizes several of the ATLAS experiment [1] benchmark analyses as of November 2012, with integrated luminosity ranging from $4.7-5 \mathrm{fb}^{-1}$ for $7 \mathrm{TeV}$ (2011) and 6-13 $\mathrm{fb}^{-1}$ for $8 \mathrm{TeV}$ (2012). In the following, the collection of simple two-body resonances: $\ell^{+} \ell^{-}, \gamma \gamma$, $j j$, and $\ell \nu[2-9]$ and diboson resonances: $W W \rightarrow \ell \nu \ell^{\prime} \nu^{\prime}$ and $Z Z \rightarrow \ell \ell j j[10,11]$ will be discussed. The two types of resonances are covered by a spectrum of models that are described in detail in the ATLAS publications [2-11] ${ }^{1}$. The models discussed by these analyses are: Heavy gauge bosons, $Z^{\prime}[2,3]$ and $W^{\prime}[7,9]$, from higher symmetry (e.g. $E_{6}$ ) breaking or from a sequential extension of the SM (denoted as SSM), Composite models for quarks, e.g. $q^{*}$, with substructure scale $\Lambda$ [6-8], Randal-Sundrum (RS) gravitons, $G^{*}$ and $G_{\text {bulk }}^{*}$, from warped extra dimensions (EDs) $[3-5,10,11]$, Low-scale string resonances (SR) with large EDs [7], $\mathrm{TeV}^{-1}$ Kaluza-Klein (KK) excitations of $\gamma / Z$ [3], Technicolor (TC) and minimal walking TC (MWT) [3], Torsion (TS) [3] and chiral bosons $Z^{*} / W^{*}[3,9]$, Color octet scalars (s8) [7], Quantum black holes (QBH) [7], ADD gravitons [5], contact interactions (CI) $[5,7]^{2}$.

The connection between the different experimental signatures and the model predictions is given in table 1 .

\footnotetext{
a e-mail: hod@cern.ch

${ }^{1}$ A comprehensive description of each model can be found in these publications and thereby references.

${ }^{2}$ The QBH, ADD and CI models predict non resonant signatures but these can be examined by looking on the same experimental signatures as for resonant models.
}

Table 1. The experimental signatures studied by the ATLAS analyses presented here and the corresponding interpretations, within these analyses. The first three rows correspond to $8 \mathrm{TeV}$ analyses (the rest for $7 \mathrm{TeV}$ ).

\begin{tabular}{c|c|c}
\hline Signature & Models & Publication \\
\hline$\ell^{+} \ell^{-}$ & $Z^{\prime}$ & {$[2]$} \\
$j j$ & $q^{*}$ & {$[6]$} \\
$\ell \ell j j(Z Z)$ & $G_{\mathrm{bulk}}^{*}$ & {$[11]$} \\
\hline$\ell^{+} \ell^{-}$ & $Z^{\prime}, G^{*}, \mathrm{TC}, \mathrm{KK}, \mathrm{TS}, Z^{*}$ & {$[3]$} \\
$j j$ & $q^{*}, \mathrm{QBH}, W^{\prime}, \mathrm{CI}$, Strings & {$[7]$} \\
$\ell \nu$ & $W^{\prime}, W^{*}$ & {$[9]$} \\
$\ell \nu \ell^{\prime} \nu^{\prime}(W W)$ & $G^{*}, G_{\mathrm{bulk}}^{*}$ & {$[10]$} \\
$\gamma \gamma$ & $\mathrm{RS}, \mathrm{ADD}$ & {$[5]$} \\
\hline
\end{tabular}

This note is divided into two parts, one dealing with simple two-body resonances and the second dealing with diboson resonances. The separation within each part is signaturewise where for several signatures, there is an overlap with respect to the models interpretation of the limit on the models parameters.

\section{Simple two-body resonances}

\subsection{Dilepton}

The dilepton signature in this analysis [2-4] is very clean, making possible the identification or exclusion of high mass resonances. Pairs of high- $p_{T}$, isolated $e$ or $\mu$ 


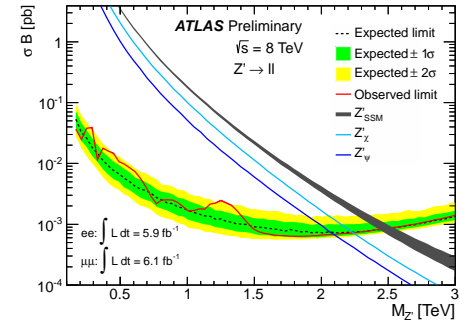

Figure 1. 95\% CL limits on $\sigma B\left(Z^{\prime} \rightarrow \ell \ell\right.$ ) (cross section times branching fraction) vs. $M_{Z^{\prime}}$ for several $Z^{\prime}$ models [2].

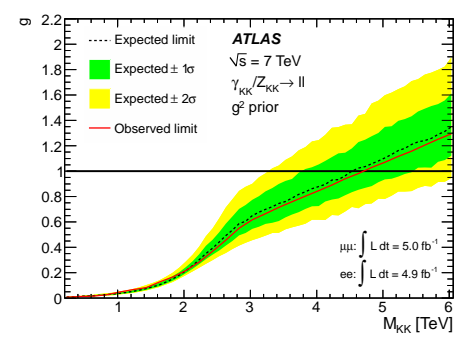

Figure 2. 95\% CL limit on the KK couplings strength, $g$, vs. $M_{\mathrm{KK}}$. The strength $g$ is given with respect to the SM partners $(\gamma / Z)$ couplings [3].

are selected, where the backgrounds are $\gamma / Z$ (estimated at NNLO in this analysis), dibosons, $t \bar{t}$, multijet and $W+$ jets. All backgrounds but the $W+$ jets are estimated from Monte Carlo (MC) while the $W+$ jets (used in the ee channel only) is estimated from the data. The $\gamma / Z$ contribution can be treated as a part of the signal as it may interfere with the new physics part, as in the KK case, or as a part of the backgrounds if it does not, as in the RS graviton case. However, in cases where the interference with the new physics amplitude is very small, as in the $Z^{\prime}$ case, it can be safely neglected and thus the $\gamma / Z$ contributions can be treated as a part of the backgrounds. The sum of backgrounds is normalized to the $Z$ peak, in the range 70-110 GeV, to cancel out mass-independent uncertainties. The remaining dominant uncertainties are $20 \%$ from theory and $21 \%$ from the ee background estimation method. The data are found to be consistent with the backgroundonly hypothesis with $p$-values $8.6 \%(e e)$ and $69 \%(\mu \mu)$. The benchmark model for this analysis is the sequential SM $Z^{\prime}$. In the $8 \mathrm{TeV}$ analysis [2] it was the only model considered along with several $E_{6}$-inspired $Z^{\prime}$ s, where the limits on these are always lower than for the $Z_{\mathrm{SSM}}^{\prime}$.

In figures 1 and 2, two of the combined (ee and $\mu \mu$ channels) mass limits derived from the dilepton invariant mass distributions are presented. In figure 2, the limit on $M_{\mathrm{KK}}$, the mass of the first KK excitation of the $\gamma / Z$ particles, is the highest to date - almost $1 \mathrm{TeV}$ higher than previous limits obtained from indirect measurements. It is also the highest direct limit set on a resonance mass in ATLAS. Table 2 lists several representative limits on the parameters of various models, obtained from the two
Table 2. A collection of representative limits on the parameters of various models, as derived from the dilepton mass distributions. The limit in the first row $\left(Z_{\mathrm{SSM}}^{\prime}\right)$ was calculated also for $8 \mathrm{TeV}$ [2], where the number in parentheses corresponds to $7 \mathrm{TeV}$ [3]. The rest of the results are for $7 \mathrm{TeV}$ only [3].

\begin{tabular}{l|l}
\hline Model & Observed limit $[\mathrm{TeV}]$ \\
\hline$Z_{\mathrm{SSM}}^{\prime}$ & $M_{Z_{\mathrm{SSM}}^{\prime}} \geq 2.49(2.22)$ \\
\hline$\gamma_{\mathrm{KK}} / Z_{\mathrm{KK}}$ & $M_{\mathrm{KK}} \geq 4.71$ \\
$Z^{*}$ & $M_{Z^{*}} \geq 2.20$ \\
$G^{*}$ & $M_{G^{*}} \geq 2.16$ for $k / \tilde{M}_{\mathrm{Pl}}=0.1$ \\
Torsion & $M_{\mathrm{TS}} \geq 2.29$ for $\eta_{\mathrm{TS}}=0.2$ \\
MWT & $M_{A} \geq 1.56$ for $\tilde{g}=2$ \\
\hline \multirow{2}{*}{ Technicolor } & $M_{\rho_{\mathrm{T}} / \omega_{\mathrm{T}}} \geq 0.85$ \\
& for $M_{\rho_{\mathrm{T}}}-M_{\pi_{\mathrm{T}}}=M_{W}$ \\
\hline \multirow{2}{*}{ Minimal $Z^{\prime}$} & $M_{Z_{\min }^{\prime}} \geq 1.11-2.10$ \\
& for $\gamma^{\prime}=0.2, \theta \in[0 . \pi]$ \\
\hline
\end{tabular}

dilepton analyses. The dilepton signature can be utilized to search also for non-resonant signatures predicted by the set of CI models [4].

\subsection{Diphoton}

In this analysis [5], the $\gamma \gamma$ results are combined with the $\ell \ell$ results [3] and [4] (see section 2.1) for the applicable models ( $7 \mathrm{TeV}$ only). This is another clear signature where one looks on the invariant mass of the two highest$E_{T}(>25 \mathrm{GeV})$ isolated photons in an event. The benchmark model in this analysis is the RS graviton. The backgrounds for this analysis are divided into two: (a) irreducible: SM $\gamma \gamma$ (estimated at NLO from MC), and (b) reducible: $\gamma j, j \gamma$ and $j j$ with one or two jets faking photons (estimated from the data). The first two options differ by the identity of the leading- $p_{T}$ object - the photon or the jet (fake photon). The sum of backgrounds is normalized in the control region $\left(142<m_{\gamma \gamma}<409 \mathrm{GeV}\right)$ to cancel out mass-independent uncertainties. The reducible background is extrapolated to high masses using a smooth function $f\left(m_{\gamma \gamma}\right)=p_{1} \cdot m_{\gamma \gamma}^{p_{2}+p_{3}} \log m_{\gamma \gamma}$. The dominant uncertainties in this analysis are: $9 \%$ (photon identification and isolation), 5-15\% (mostly due to parton distribution functions (PDFs)). The data are found to be consistent with background-only hypothesis with $p$-value $86 \%$.

Figure 3 shows the limit on the RS graviton mass for few values of the dimensionless couplings, $\kappa / \tilde{M}_{\mathrm{Pl}}$. Combining the result with dileptons yields a limit of 1.03 and $2.23 \mathrm{TeV}$ on the RS graviton mass, for $\kappa / \tilde{M}_{\mathrm{Pl}}=0.01$ and 0.1 respectively. The diphoton channel can be utilized to search also for non-resonant signatures such as those predicted by the ADD model for example. In that case, the limit on the number of ADD signal events in the search region at $m_{\gamma \gamma}>1217 \mathrm{GeV}$ is 7.21 and the lower limits on the ultraviolet cutoff of the $\mathrm{KK}$ spectrum, $M_{S}$, range between 2.79 and $4.18 \mathrm{TeV}$ depending on the number of EDs and the theoretical formalism (see [4]). 


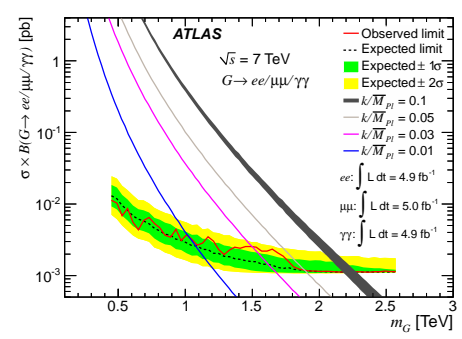

Figure 3. 95\% CL limits on $\sigma B\left(G^{*} \rightarrow \mu \mu+e e+\gamma \gamma\right)$ vs. $M_{G^{*}}$ for few values of $\kappa / \tilde{M}_{\mathrm{Pl}}[5]$.

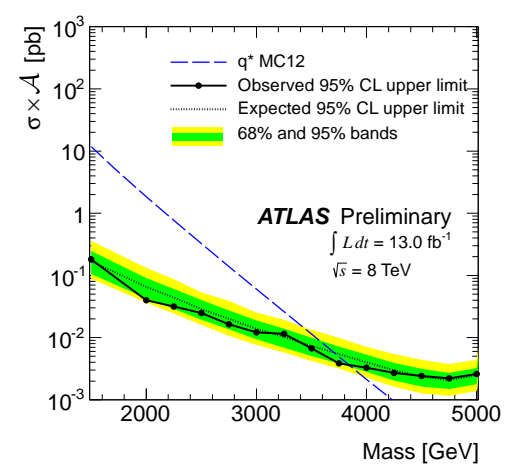

Figure 4. $95 \%$ CL limits on $\sigma \mathcal{A}\left(q^{*}\right)$ vs. $M_{q^{*}}$ [6].

\subsection{Dijet}

In this analysis [6-8], the mass and angular distribution of the two most high- $p_{T}$ jets in an event are used. The benchmark model used is the excited quark, $q^{*}$. The analysis takes advantage of the fact that the dominant $t$-channel QCD interactions lead to angular distribution that peak at small scattering angles while the signal is expected to be more isotropic. Therefore, the angular distributions are used (in addition to the dijet mass distribution). A data driven background estimation leading to the $m_{j j}$ spectrum is parametrized by the function $f(x)=p_{1}(1-x)^{p_{2}} x^{p_{3}+p_{4} \ln (x)}$ where, $x \equiv m_{j j} / \sqrt{s}$, while the background estimate for the angular analyses relies on QCD MC. The dominant uncertainties for the mass analysis are: $4 \%$ for $p_{T}^{\text {jet }}>1 \mathrm{TeV}$ from the jet energy scale (JES) and 3.6-3.9\% from the luminosity uncertainties. The dominant uncertainties for the angular analysis are: $<8 \%$ due to NLO QCD renormalization and factorization scales, and $<15 \%$ due to JES. The angular analysis (7 TeV) employs ratio observables called $\chi$ and $F_{\chi}$ (see [7] for definition) to reduce its sensitivity to systematic uncertainties (JES, PDFs, luminosity) and is more sensitive to non-resonant signals than the mass analysis. The data are found to be consistent with background-only hypothesis with $p$-value $61 \%$.

Figures 4 and 5 show the limit on the cross section times acceptance vs. the resonance mass for $q^{*}$ and for a generic Gaussian model (see [7, 8] on how to interpret the Gaussian limits). Table 3 lists several representative

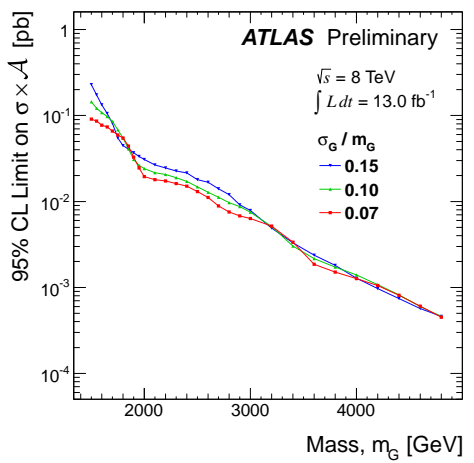

Figure 5. 95\% CL limits on $\sigma \mathcal{A}\left(\right.$ Gaus $\left.^{\prime}\right)$ vs. $M_{\text {Gaus' }^{\prime}}[6]$.

Table 3. A collection of representative limits on the parameters of various models derived from the dijet mass and angular distributions. The limit in the first row $\left(q^{*}\right)$ was calculated also for $8 \mathrm{TeV}$ [6], where the number in parentheses corresponds to $7 \mathrm{TeV}$ [7]. The rest of the results are for $7 \mathrm{TeV}$ only [7]. The last two rows correspond to limits on non-resonant models, obtained from the angular distribution only [7].

\begin{tabular}{l|lc}
\hline Model & \multicolumn{2}{|c}{ Observed limit [TeV] } \\
& from $m_{j j}$ or $\chi$ & from $F_{\chi}$ \\
\hline$q^{*}$ & $M_{q^{*}} \geq 3.84(2.83)$ & - \\
\hline Color octet scalar & $M_{\mathrm{s} 8} \geq 1.86$ & - \\
$W^{\prime}$ (SM couplings) & $M_{W^{\prime}} \geq 1.68$ & - \\
String resonances & $\mathrm{SR}$ scale $\geq 3.61$ & - \\
\hline QBH for 6 (2) EDs & $M_{D} \geq 4.11$ & $4.03(3.71)$ \\
CI (destructive int') & $\Lambda \geq 7.6$ & 7.6 \\
\hline
\end{tabular}

limits obtained from both the mass and the angular distribution analyses.

\subsection{Charged lepton and a neutrino}

This analysis [9] requires exactly one isolated, high- $p_{T}$ muon or electron with $p_{T}>25$ and $85 \mathrm{GeV}$ respectively. A missing transverse energy $\left(E_{T}^{\text {miss }}\right)$ with same thresholds is also required. The kinematic variable used to identify a $W^{\prime} / W^{*}$ signal is the transverse mass, $m_{T}=$ $\sqrt{2 p_{T}^{\ell} E_{T}^{\text {miss }}\left(1-\cos \phi_{\ell \nu}\right)}$. These two signals are distinguishable with respect to both the $m_{T}$ and the angular distributions. The dominant backgrounds are: SM $W$ bosons (estimated at NNLO), $Z \rightarrow \ell \ell$ with one lepton not reconstructed, $\tau$ 's from $W / Z$ and diboson production, $t \bar{t}$ and single-top production and QCD where a hadron decays semileptonically or a jet is misidentified as an electron. The dominant uncertainties are: $12 \%$ (cross sections) and $<5 \%$ (experiment). The data are found to be consistent with the background-only hypothesis.

Figure 6 shows the limit on the cross section times branching fraction vs. $M_{W^{\prime}}$ where the corresponding scenario for the $W^{*}$ model is comparable. For the $W^{\prime}$ theory curve in figure 6 , no interference with $\mathrm{SM} W$ is taken. This is again a sequential SM scenario. For the $W^{*}$ limit, 


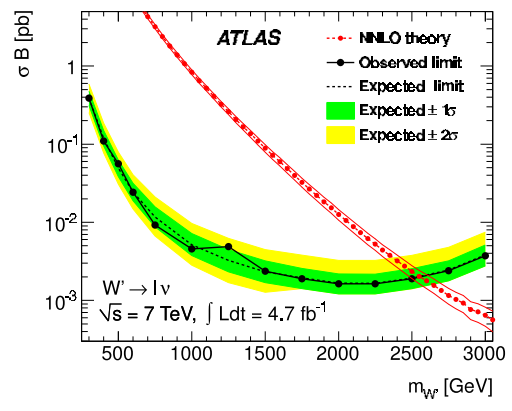

Figure 6. 95\% CL limits on $\sigma B\left(W^{\prime}\right)$ vs. $M_{W^{\prime}}$ for the combination of the $e$ and $\mu$ channels [9].

the $W^{*}$ is taken with $q$ and $g$ coupling strengths normalized to reproduce the $W^{\prime}$ width. The limit on $M_{W^{\prime}}\left(M_{W^{*}}\right)$ is $2.55(2.42) \mathrm{TeV}$.

\section{Diboson resonances}

\subsection{WW resonance $\left(\ell \nu \ell^{\prime} \nu^{\prime}\right)$}

In this analysis [10], the signature is less pronounced than for the simple two-body resonances because of the subsequent decay of the $W$ bosons produced in the hard interaction. More over, the presence of two $W$ particles implies a large and non-resolved $E_{T}^{\text {miss }}$. In this analysis, one requires exactly two oppositely-charged isolated, high- $p_{T}(>25 \mathrm{GeV})$ leptons, and a large $E_{T}^{\text {miss }}(>30,60$ and $65 \mathrm{GeV}$ for $e \mu$, ee and $\mu \mu$ ). In order to reduce $Z$ and top backgrounds, it is required that $m_{\ell \ell}>106 \mathrm{GeV}$ and events with $b$-jets are discarded (the tagging efficiency of $b$-jets is $\epsilon_{b}^{\mathrm{tag}} \sim 85 \%$ ). The $W W$ transverse mass, $m_{T}^{W W}=$ $\sqrt{\left(\sum_{i=1}^{2} p_{T}^{\ell_{i}}+E_{T}^{\mathrm{miss}}\right)^{2}-\sum_{k=x, y}\left(\sum_{i=1}^{2} p_{k}^{\ell_{i}}+E_{k}^{\mathrm{miss}}\right)^{2}}$, is used as a discriminant variable. The RS graviton is again the benchmark model. However, since this analysis concentrates on the possibility of decay into a pair of heavy $W$ particles, the sensitivity for another theoretical variation of the ordinary RS model is greater. In the "bulk" RS graviton, $G_{\text {bulk }}^{*}$ model, where the ED setup is slightly different than for the ordinary RS model, the graviton has enhanced couplings to the heavier particles, leading to large branching fractions for these states, e.g. $\operatorname{Br}\left(G_{\text {bulk }}^{*} \rightarrow W W\right) \sim 15 \%$. The dominant backgrounds are: SM $W W$ (estimated at NNLO) and $W Z / Z Z$ with only two reconstructed leptons, $W \gamma$ where the $\gamma$ is reconstructed as a lepton, $t \bar{t}$ (estimated at NLO, with zero $b$-jets) and single-top (zero $b$-jets), $W / Z+$ jets and QCD multi-jet production (both estimated from data). The main uncertainties are: $<5 \%$ (due to muon resolution correction), 2 $9 \%$ (JES), 3.5\% ( $E_{T}^{\text {miss }}$ energy scale), 6-21\% $\left(\epsilon_{b}^{\text {tag }}\right.$ estimation) and 5-10\% (cross sections) and 10-30\% ( $W+$ jets estimation). The data are found to be consistent with background-only hypothesis with $p$-value $>8 \%$.

Figure 7 shows the limit on the cross section times branching fraction vs. $M_{G_{\text {bulk }}^{*}}$. The limits on the ordinary RS and the "bulk" RS graviton mass are 1.23 and $0.84 \mathrm{TeV}$ respectively.

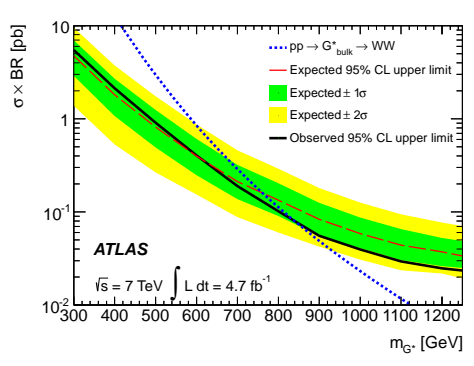

Figure 7. $95 \% \mathrm{CL}$ limits on $\sigma B\left(G_{\mathrm{bulk}}^{*} \rightarrow W W\right)$ vs. $M_{G_{\text {bulk }}^{*}}[10]$.

\section{$3.2 \mathrm{ZZ}$ resonance $(\ell \ell j j)$}

In this analysis [11], the signature is again less pronounced than for the simple two-body resonances because of the subsequent hadronic decay of one of the produced $Z$ bosons. One requires exactly two isolated, high- $p_{T}$, same flavor leptons and two high- $p$ jets (not within $\Delta R=0.3$ around a lepton). The presence of leptons reduce the multijet background with respect to the fully-hadronic final state and allow a complete kinematic reconstruction of the intermediate states. The "bulk" RS graviton is the only signal considered in this analysis. For highly boosted $Z$ bosons, the $\eta-\phi$ distance between the two quarks can be parametrized as $R_{q q} \approx 2 M_{Z} / p_{T}^{Z}$. Therefore, in resonances with masses above $\sim 900 \mathrm{GeV}$, the two quarks can fall within a $\Delta R=\sqrt{\Delta \phi^{2}+\Delta \eta^{2}}=0.4$ cone, resulting in a single reconstructed massive jet where $m_{j j}\left(m_{j}\right) \simeq$ $M_{Z}$. As a result, two signal selections must be defined, (a) resolved and (b) merged. The background modeling is tested in these two selections for the $m_{\ell \ell j j}\left(m_{\ell \ell j}\right)$ distributions respectively. Muons are required to be oppositelycharged and the $\ell \ell$ invariant mass is required to be in the range $66<m_{\ell \ell}<116 \mathrm{GeV}$ in order to ensure a $Z$ origin. The dominant backgrounds are: SM $Z+$ jets (estimated at NLO), $t \bar{t}, \mathrm{SM}$ diboson production, $W+$ jets and QCD (estimated from data). The final background is estimated by fitting the $m_{\ell \ell j j}\left(m_{\ell \ell j}\right)$ distributions in the data to a smooth function $f(x)=p_{1}(1-x)^{p_{2}} x^{-p_{3}-p_{4} \ln (x)}$ where $x \equiv m_{\ell \ell j j} / \sqrt{s}$ or $x \equiv m_{\ell \ell j} / \sqrt{s}$ for the two selections respectively. The main uncertainties are: $5 \%$ for $m_{\ell \ell j j}$ $<800 \mathrm{GeV}$ and $10-40 \%$ for $m_{\ell \ell j}$ (due to the background fit), $11-15 \%$ (overall uncertainty on the signal acceptance due to jet mass scale, luminosity, JES and ISR/FSR modeling). The data are found to be consistent with backgroundonly hypothesis.

Figure 8 shows the limit on the cross section times branching fraction vs. $M_{G_{\text {bulk }}^{*}}$ where the two signal selections are combined by showing only limits for one of them in the range where the expected limit is better. The limit on the "bulk" RS graviton mass is $0.84 \mathrm{TeV}$.

\section{Summary}

This note covers the most recent ATLAS searches for heavy resonances in 8 different analyses and for 6 different signatures. The most massive observed event in the data, 
among these analyses, has a mass of $4.69 \mathrm{TeV}$ (dijet). No significant deviation from the SM expectations is found in any of these signatures and a set of cutting-edge limits on the parameters of more than 10 models is obtained with a mass reach as high as $4.71 \mathrm{TeV}$ (on $M_{\mathrm{KK}}[3]$ ).

\section{References}

[1] ATLAS Collaboration, JINST 3, S08003 (2008)

[2] ATLAS Collaboration, ATLAS-CONF-2012-129

[3] ATLAS Collaboration, arXiv:1209.2535, accepted by JHEP (2012)

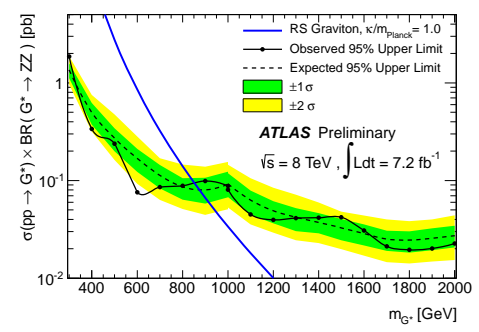

Figure 8. 95\% CL limits on $\sigma B\left(G_{\text {bulk }}^{*} \rightarrow Z Z\right)$ vs. $M_{G_{\text {bulk }}^{*}}[11]$.
[4] ATLAS Collaboration, arXiv:1211.1150, submitted to Phys. Rev. D (2012)

[5] ATLAS Collaboration, arXiv:1210.8389, submitted to Phys. Lett. B (2012)

[6] ATLAS Collaboration, ATLAS-CONF-2012-148

[7] ATLAS Collaboration, arXiv:1210.1718, submitted to JHEP (2012)

[8] ATLAS Collaboration, Phys. Lett. B708, 37 (2012)

[9] ATLAS Collaboration, arXiv:1209.4446, submitted to Eur. Phys. J. C (2012)

[10] ATLAS Collaboration, arXiv:1208.2880, submitted to Phys. Lett. B (2012)

[11] ATLAS Collaboration, ATLAS-CONF-2012-150 